\title{
Operating System Version or Upgrade Problem
}

National Cancer Institute

\section{Source}

National Cancer Institute. Operating System Version or Upgrade Problem. NCI

Thesaurus. Code C62893.

Problem associated with replacing an older operating system to an up-to-date operating system. 